\title{
Probabilistic assessment of power system mode with a varying degree of wind sources integration
}

\author{
Nariman Rahmanov ${ }^{1}$, Viktor Kurbatskiy ${ }^{2}$, Huseyngulu Guliyev ${ }^{*}$, Nikita Tomin ${ }^{2}$, Zaur Mammadov ${ }^{1}$ \\ ${ }^{1}$ Azerbaijan Scientific-Research and Designed-Prospecting Institute of Energetics, Baku, Azerbaijan \\ ${ }^{2}$ Melentiev Energy Systems Institute, 130 Lermontov str., Irkutsk, Russia
}

\begin{abstract}
At present among renewable sources the wind and solar plants have the most significant portion of power generation. Randomly changing and intermittent nature of this power leads to the stochasticity of the power grid mode, estimation of parameters of which requires application of probabilistic modeling. In the paper it is proposed an advanced algorithm of probabilistic load flow based on the development of two-point estimation method, the efficiency of which is confirmed on the basis of computational experiments and comparative analysis of the Monte Carlo simulation results. Calculations and analysis of the modeling results were carried out on standard 14-nodal scheme of IEEE and real electrical network of "Azerenerji” Grid.
\end{abstract}

\section{Introduction}

Calculation for the steady-state mode is a significant part of the total scope of researches on electric power systems (EPS) both at the design stage and during the operation of these systems $[1,2]$. In recent years, the growth of power generation from renewable energy sources and mainly due to the large share of generation capacity from wind and solar energy converters with the stochastic nature increased the uncertainty in the power system state. This circumstance has led to larger need to solve the problems of planning, analysis and system management based on stochastic modeling techniques. For the first time the probabilistic modeling of the load flows in the power system was considered in [3], in which the stochastic input variable was taken to be the load power value in one of the nodes of the system.

At that the variability of the load power was reproduced by Monte Carlo method, which led to the necessity to consider the tens of thousands and more numbers of steady states. At the beginning of the 2000s a number of authors $[4,10,11]$ proposed a simplified approach for probabilistic modeling of the load flows (PR), based on the analysis of initial data for load power values, power generation by wind turbines and relevant functions of their distribution in two given points.

Probabilistic methods of calculation and analysis of load flows (LF) in the system are based on two approaches: numerical simulation by Monte Carlo method [3-5,9,10], and analytical methods of the transformation in the form of cumulated representations [6], [7], or using of point values of probability distribution functions $[8,9]$.

In a probabilistic formulation the problem of LF assumes the initial information assignment in the form of the probability distribution function of power at the nodes of generation and load, as well as the probability of random changes in the system diagram. Presence of description of random states of EPS with given probabilistic estimates of characteristics for the system input values allows for numerical or analytical determination of the probabilistic estimates of the variables at the system output - the voltage at the nodes, power flows and power losses in the Grid.

Modeling of the probabilistic state of the system, containing wind power plants (WPP), enables to solve the problem of planning and analysis of modes, taking into account their stochastic variation.

The paper presents the results of the modeling, calculation and analysis of EPS modes at stochastic variability of power values of load and sources, as well as considering the probability of emergency shutdown of transmission lines of backbone network.

To simulate such stochastic processes the combined approach of Monte Carlo method and two-point assessment method is used. It is known that the stochastic variability of the load power is fairly well described by a normal distribution law and the MonteCarlo method is the most appropriate for its reproduction. 


\section{Probabilistic modeling of system state with wind farms}

Probabilistic assessment of system state is caused by stochastic variability of generation in the nodes, containing sources with random-intermittent power output, fluctuation of power consumption in load nodes, as well as random changes in the topology (scheme) of the system. The values of the variables of the system state are determined based on recurring solutions of steady-state equations (SSE) for various combinations of values of variables in the input. The values of the variables at the output (the result of the SSE solution) are the values of flows of active and reactive power in the lines, the voltages at the subsystems. To solve the LF problem in deterministic formulation, various methods and algorithms are developed [11-15], which are widely used in practice.

Deterministic model of the SSE is described in the following form:

$$
\begin{aligned}
& \sum_{G} P_{i}^{G}-\sum_{D} P_{i}^{D}=U_{i} \sum_{j=1}^{n} U_{j}\left(G_{i j} \cos \delta_{i j}+B_{i j} \sin \delta_{i j}\right) \\
& \sum_{G} Q_{i}^{G}-\sum_{D} Q_{i}^{D}=U_{i} \sum_{j=1}^{n} U_{j}\left(G_{i j} \sin \delta_{i j}+B_{i j} \cos \delta_{i j}\right)
\end{aligned}
$$

where $P_{i}^{G}, P_{i}^{D}$ и $Q_{i}^{G}, Q_{i}^{D}$ - active and reactive powers of generation and consumption respectively in $i$ - node;

$U_{i}, U_{j}$ - voltage value in $i$ and $j$ nodes respectively;

$G_{i j}, B_{i j}$ - active and reactive conductance;

$\delta_{i j}$ - phase shift between $U_{i}$ and $U_{j}$ vectors.

At random nature of variability of input variables, specified as a probability vector

$$
X=\left\lfloor P_{i}^{G}, P_{i}^{D}, Q_{i}^{G} Q_{i}^{D} \ldots \ldots\right\rfloor
$$

the SSE will be represented in the form of equation set combination (1), (2) with the values of the input variables defined as a series of random sample or distribution function of these variables. In this case, the stochastic system state is described in the form of relationship of randomly changing values of power overflows in the network and the voltages in the nodes from the random values of powers in the nodes of generation and load respectively.

$$
Y=F(X)
$$

where

$$
\left.Y=\left\lfloor P_{i j}, Q_{i j}, \delta_{i j}, U_{i} \ldots . .\right\rfloor\right\rfloor
$$

For wind turbine equipped with the asynchronous generator, the probabilistic model for generation of active and reactive power can be represented as the relationship from wind speed. At simplified representation of the asynchronous generator scheme, such relationship has the following form:

$$
\begin{gathered}
P_{w i}^{G}=\frac{-s R_{2}}{R_{2}^{2}+s^{2} X_{i}^{2}} \cdot U_{i}^{2}= \\
=\left\{\begin{array}{lc}
0, & v \leq v_{c i} \\
P_{n m} \cdot \frac{v-v_{c i}}{v_{H}-v_{c i}}, & v_{c i}<v \leq v_{H} \\
P_{n m}, & v_{H}<v \leq v_{c o} \\
0, & v>v_{c o}
\end{array}\right. \\
Q_{m i}^{G}=\frac{s X}{R_{2}} P_{w i}
\end{gathered}
$$

where $P_{m i}^{G}, Q_{m i}^{G}$ - capacity of production of active and reactive power of wind farm connected to $i$ - node of the system; $S$-sliding of wind turbine; $R$ - active resistance of rotor of asynchronous generator; $X$ - the sum of dissipation reactances of stator and rotor; $v_{c i}$ the wind speed at which the asynchronous generator starts; $v_{c o}$ - the wind speed at which the wind turbine is switched off $; v_{H}$ - the wind speed, which provides a nominal power output.

In equation (5) the influence of the stochastic variability of wind speed on the power generation value of wind turbine $P_{w i}$ appears through the stochastic variability of the sliding value of the asynchronous generator. In this connection, during the probabilistic assessment of system state with stochastically varying power output of asynchronous wind turbines, one of the state parameters is the sliding value. At that it should be noted that the values of the generation of active and reactive power of the wind turbine will be random variables dependent on the stochastically changing wind speed.

Analysis of probabilistic LF in the electrical system will be performed based on modeling using the Monte Carlo method and analytical method of evaluation of the curve of the probability density function by two points (2TOM method).

The Monte Carlo method is the most commonly used due to the simplicity of the modeling implementation regardless of the number of random variables defining the system state, its complexity and the "output variable - input variable" nonlinear relationship. At the same time, the Monte Carlo method requires large expenditures for computing resources to conduct repetitive calculations carried out 10 thousand or more times. We will not consider in detail the algorithm of the Monte Carlo method because it is widely used in power industry $[8,9]$. 
2TOM method gives the opportunity for estimating the stochastic state of the system by determining the values of the variables or their distributions at the model output using the specified values of the variables or their distributions at the output at two points of the current state of the model.

For probabilistic models of LF, described in general form by equations (1), (2), (4), the state estimation by 2TOM method takes as input data the values of generation and load (variables $x_{i}$ ) that vary with the probability of distribution function chosen for two points, located on opposite sides of the mean value. At that, as a practice of this method application shows, for the function characterization it is enough to specify three moments - mean, standard deviation and asymmetry coefficient.

The algorithm for calculation of distribution probability functions and moments of random variables characterizing the state of load flows - voltages in nodes and power flows in the lines -consists of the following stages [11]:

1. The choice of the number of fuzzy variables $n$ For 14-nodal IEEE test scheme $\mathrm{n}=3$ is selected - one node of the load, which is taken into account by stochastic model of the power size; one node with connected wind farm and one linear element (transmission line) with a given probability of emergency shutdown.

2. It is assumed that the mathematical expectation and standard deviation of the state variables at the output are equal to zero

$$
E(Y)=0 ; \quad E\left(Y^{2}\right)=0
$$

3. It is assumed that the number of input variables that are located above and below the mean value is $k=1$.

4. Determination of concentration point $\varepsilon_{k, 1}$ and $\varepsilon_{k, 2}$ and concentration probability $p_{k, 1}$ and $p_{k, 1}$ for the variables above and below their mean value

$$
\begin{gathered}
\varepsilon_{k, 1}=\sqrt{n} \\
\varepsilon_{k, 1}=-\sqrt{n} \\
p_{k, 1}=p_{k, 2}=\frac{1}{2 n}
\end{gathered}
$$

5. Determination of two values of input variable concentration

$$
\begin{aligned}
& \chi_{k, 1}=\mu_{x, k}+\varepsilon_{k, 1} \cdot \sigma_{x, k} \\
& \chi_{k, 2}=\mu_{x, k}+\varepsilon_{k, 2} \cdot \sigma_{x, k}
\end{aligned}
$$

where $\mu_{x, k}$ и $\sigma_{x, k}-$ mean and standard deviation of variable $x_{k}$, respectively.

6. Calculation of LF at input variable values for both concentration positions $\chi_{k, i}$.

7. Calculation of new values of moments for random output variables $Y$.

$$
\begin{gathered}
E(Y) \cong \sum_{k=1}^{n} \sum_{i=1}^{2}\left(p_{k, i} h\left(\left[\mu_{x, 1} \cdots x_{k, i} \cdots \mu_{x, n}\right]\right)\right) \\
E\left(Y^{2}\right) \cong \sum_{k=1}^{n} \sum_{i=1}^{2}\left(p_{k, i} h\left(\left[\mu_{x, 1} \cdots x_{k, i} \cdots \mu_{x, n}\right]\right)^{2}\right)
\end{gathered}
$$

8. Calculation of mean and standard deviation

$$
\begin{aligned}
& \mu_{Y}=E(Y) \\
& \sigma_{Y}=\sqrt{E\left(Y^{2}\right)-\mu_{Y}^{2}}
\end{aligned}
$$

9. Calculations of probabilistic characteristics for the input variable $x_{2,1}(k=2)$.

\section{Modeling results}

Estimation experiments were conducted at 14- nodal test scheme of IEEE and real scheme of Azerenerji system with 219 nodes, in which the power values of load and generation nodes were considered as fuzzy random variables, as well as telemetry data, defining the probability of a normal (emergency) status of the monitored lines.

Probabilistic calculations for load flows were performed using ETAP-5 software complex.

Model of 14-nodal IEEE scheme. Single-line diagram of the system is shown in Fig.1, in which the $50 \mathrm{MW}$ wind farm is connected to the system at node 8 . It is assumed that the load powers at the nodes are stochastic with normal distribution function of probability density. The probability distribution of power generation of wind farm is determined based on the sample data of power values computed according to (5).

Let's perform a comparative analysis of results of modeling of electrical system modes for the following options of scheme state (Table1).

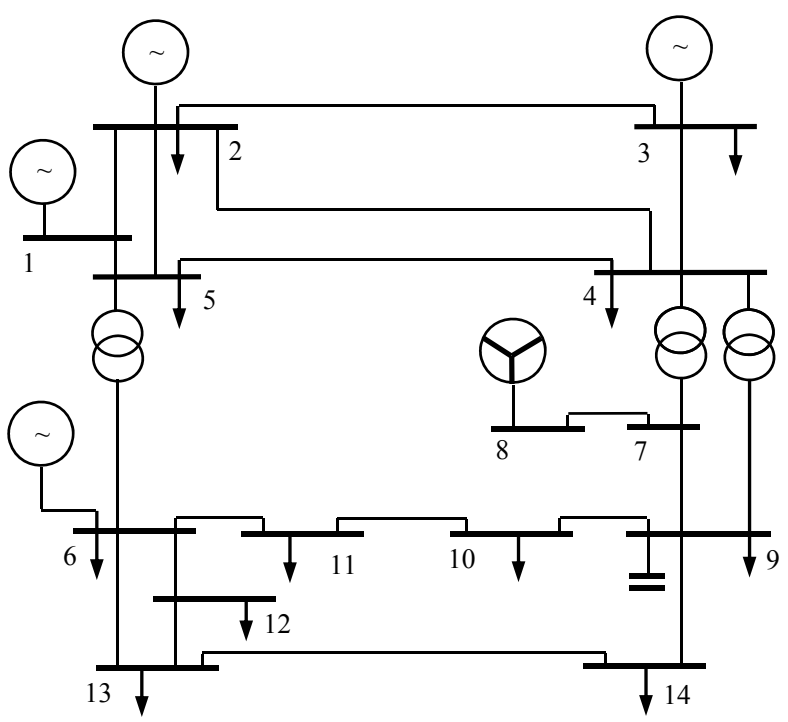

Fig. 1. Test IEEE scheme 
Table 1. Options of stochastic states of power output of WPP, load capacities and the current scheme state probability

\begin{tabular}{|c|c|c|c|}
\hline $\begin{array}{c}\text { System } \\
\text { state } \\
\text { options }\end{array}$ & \multicolumn{2}{|c|}{ Model of load and wind farm } & $\begin{array}{c}\text { Network } \\
\text { topology } \\
\text { state }\end{array}$ \\
\hline 1 & fixed & $\begin{array}{c}\text { With changing } \\
\text { generation (for } \\
\text { periods with } \\
\text { constant wind } \\
\text { speeds) }\end{array}$ & $\begin{array}{c}\text { Normal } \\
\text { complete } \\
\text { scheme }\end{array}$ \\
\hline 2 & fixed & $\begin{array}{c}\text { Stochastically } \\
\text { changing }\end{array}$ & $\begin{array}{c}\text { Normal } \\
\text { complete } \\
\text { scheme }\end{array}$ \\
\hline 3 & $\begin{array}{c}\text { Stochastically } \\
\text { changing }\end{array}$ & $\begin{array}{c}\text { Stochastically } \\
\text { changing }\end{array}$ & $\begin{array}{c}\text { Normal } \\
\text { complete } \\
\text { scheme }\end{array}$ \\
\hline 4 & $\begin{array}{c}\text { Stochastically } \\
\text { changing }\end{array}$ & $\begin{array}{c}\text { Stochastically } \\
\text { changing }\end{array}$ & $\begin{array}{c}\text { Probability } \\
\text { of PTL } \\
\text { outage }\end{array}$ \\
\hline
\end{tabular}

The calculations of the indicators, that determine the state of the system under study, are performed for each option. As it is seen from the analysis of the results, with the increase of stochasticity of power change, the greater variability is characteristic for the standard deviation values of the state variables. The increasing uncertainty of the input variables leads to an increase in stochasticity of state variables.

Fig. 2 and Fig. 3 show curves of integral functions of the probability distribution of voltages in the nodes 7 and 14 in the absence and connection of wind farm to the system (the second option of the scheme state).

From the comparison of the distribution characteristics one can see that the voltage fluctuation in the node 7 is the most significant compared to similar fluctuations in other nodes due to the remoteness of these nodes from the wind farm connection point.

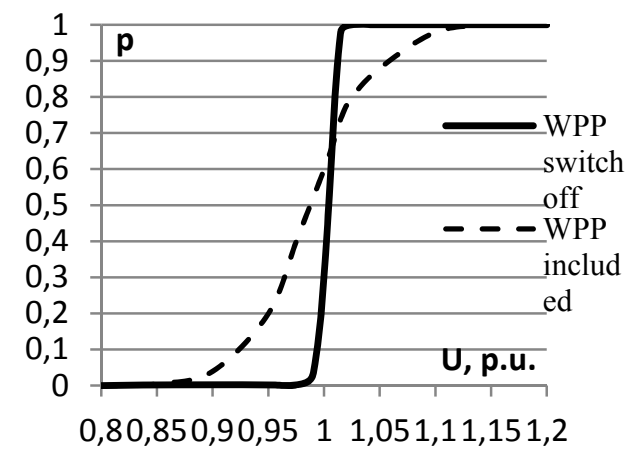

Fig.2. Cumulative distribution function of voltage probability at node 7

As can be seen from Fig. 2 and 3, when the WPP is connected, the voltage values in the nodes have random nature of the variability of probability distribution (shown in dash curve), which differs from the corresponding characteristic in the absence of the wind farm in the scheme.

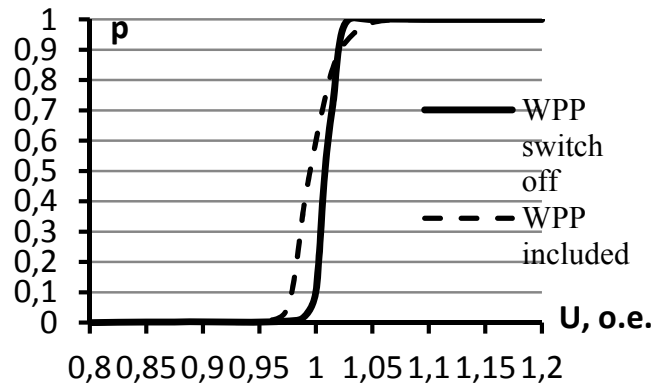

Fig.3. Cumulative distribution function of voltage probability at node 14

Model of "Azerenerji" real scheme. For the estimation of the influence of established power volume of WPP and the value of the random variation of power generation on the parameters of the electrical system state, the investigations were carried out on the model of real scheme of "Azerenerji". Estimation experiments were carried out on scheme models with connected WPP with capacity of $50 \mathrm{MW}$ and $100 \mathrm{MW}$ respectively. Fragment of the "Azerenerji" Grid with WPP connected to 330/220/110 kV "Yashma" substation is shown in the Fig.4. The system is presented in the form of two connected parts. To one of them a wind farm is connected, consisting of FL $2500 / 90$ type wind turbines with the capacity of $10 \times 2,5$ MW.

Technical parameters of FL -2500/90 DFİG type wind farm are as follows: rated power - $2500 \mathrm{\kappa W}$; wind speed at WPP initiation $\left(v_{c i}\right)-3,5 \mathrm{~m} / \mathrm{sec}$; rated wind $\operatorname{speed}\left(v_{r}\right)-13 \mathrm{~m} / \mathrm{sec}$; maximum wind speed $\left(v_{c o}\right)-25$ $\mathrm{m} / \mathrm{sec}$; output AC voltage $-690 \mathrm{~V}$.

According to Table 1, the modeling of the probabilistic LF for all four options of the system state was performed twice - for the scheme with $50 \mathrm{MW}$ and 100 MW WPP.

Analysis of modeling results shows that with the increase of generation power from the wind farm the dispersion of the voltage probability distribution in the nodes increases. The voltage distribution curves on $220 / 110 \mathrm{kV}$ "Mushfig" SS with the capacity of wind power in the system $50 \mathrm{MW}$ and $100 \mathrm{MW}$ are illustrated as example in Fig. 5 and 6. As can be seen, the growth of wind power increases the stochasticity of the system state that indicates the difference of its parameters from the same parameters for the scheme without WPP.

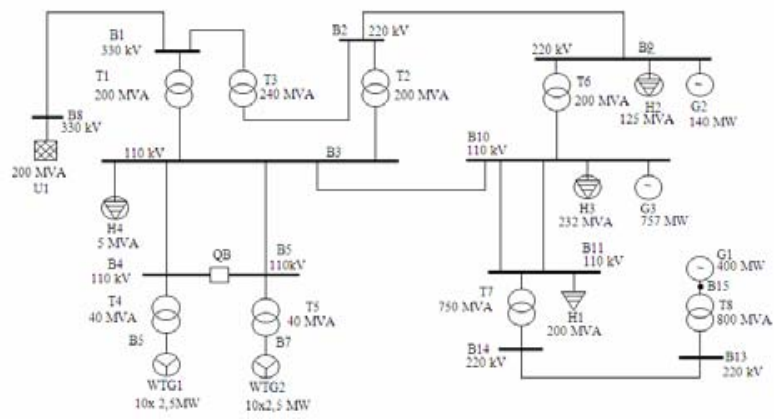

Fig.4. Simplified diagram of system under 


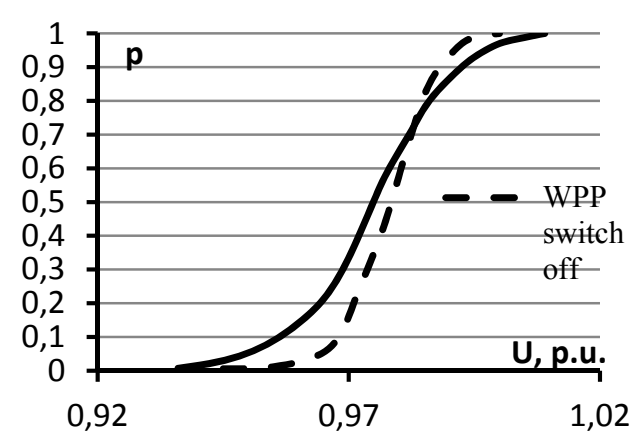

Fig.5. Cumulative distribution function of voltage probability at node B3 of "Azerenerji" scheme

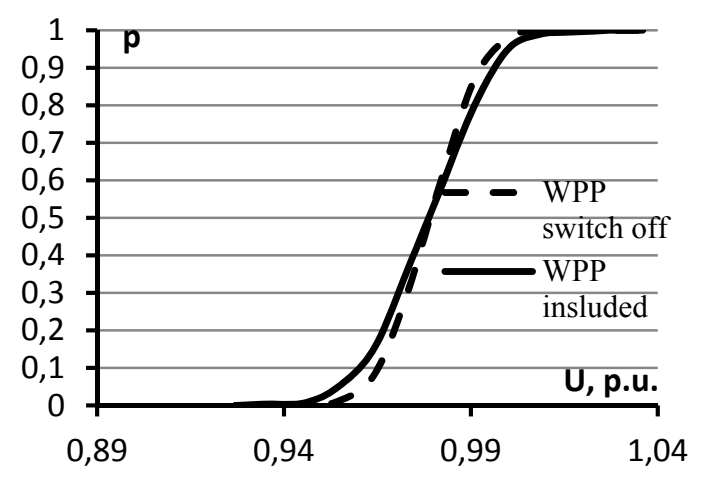

Fig.6. Cumulative distribution function of voltage probability at node B9 of "Azerenerji" scheme

\section{Conclusion}

Growth of capacity of WPP integrated to the grid power leads to the need for analysis and control of system mode based on probabilistic modeling that takes into account the stochasticity of the system state caused by WPP work. The paper presents the results of probabilistic LF modeling at different proportion of wind power integrated in the power system, assesses the probabilistic system state - voltage probability distribution in the nodes of the electrical network, power flows in the lines and the variability of the loss values. It is shown that the growth of the connected capacity of WPP can significantly change the probabilistic assessment of voltage in the network.

The model is proposed for calculation of power production by wind turbine equipped with a asynchronous generator. During the studies it is found out that when modeling the probability LF in the power system with integrated wind turbines, the stochastic variability of load, as well line failure flows in the calculated interval should be taken into account.

In the future it is necessary to develop and improve methods and tools for power grid management at stochastic changes of the state caused by renewable sources.

\section{References}

1. Electrical systems. Mathematical problems of power engineering (M: Higher school, 1981.- 288 p).

2. A. Gamm, Probabilistic methods of calculation of modes of electric power systems (Bratsk, BrRI. - 1990. - 91 p).

3. B. Borkowska, IEEE Transactions, Vol. PAS 93, No.3, pp. 752-759. (1974).

4. P. Zang, S.T. Lee, IEEE Trans. Power apparatus and Systems. February 2004, Vol. 19.- pp. 676-682.

5. P. Jorgensen, J. Christensen, J. Tande, Proc. 8th International Conf. Harmonics and Quality of Power, vol. 2.- pp. 1146-1151, Athens, Greece. (1998).

6. N. Rahmanov, H. Guliyev, International scientific seminar named after Rudenko Y. Methodical questions of research of reliability of large energy systems: Reliability of liberalized energy systems: Collection of research papers. Issue 65, St. Petersburg, pp.- 511-516 (2015).

7. A. Hashimov, H. Rahmanov, H. Guliyev, R. Rahmanov, A. Mustafayev, International scientifictechnical journal, Minsk, № 6, pp.- 519-528. (2016).

8. El - Khattab, Y.G. Hegazy and M.M. Salama, IEEE Transactions on Power Systems, Vol. 21, No. 2.pp. 524-532 (2006).

9. M. Basil, A. Jamieson, 16 th North Sea flow measurement workshop, Gleneagles, -pp. 1-10. (1998).

10. C.L. Su, IEEE Trans. Power Syst., Vol. 20, No. 4.- pp. 1843-1851. (2005).

11. G. Verbic, C.A. Canizures, IEEE Trans. Power Syst., Vol. 21, No. 4.- pp. 1883-1893. (2006).

12. B. Stott, Proceedings of the IEEE. Vol. 2, No. 7,-pp. 916-929. (1974).

13. S. Yuferev, A.V.Pazderin, Proceedings of the IASTED International Conference Power and Energy Systems (Asia PES 2013), April 10-12, 2013 Phuket, Thailand.

14. B. Ayuyev, V. Davydov, P. Yerokhin, V. Neuytin, Computational models of load flow in electrical systems. (M., Flint, Science, 2008).

15. O. Voytov, Y. Popova, L. Semenova, Electricity, №3, - pp. 19-26. (2013).

16. Handbook on probability theory and mathematical statistics (M.: Science. Chief editional board of physical and mathematical literature, 1985. $640 \mathrm{p})$. 